\title{
Efficacy of First-line Chemotherapy Affects the Second-Line Setting Response in Patients with Advanced Non-Small Cell Lung Cancer
}

\author{
Wa Cao ${ }^{1 \&}$, Ai-Wu Li ${ }^{2 \&}$, Sheng-Xiang Ren², Xiao-Xia Chen², Wei Li ${ }^{2}$, Guang-Hui \\ $\mathrm{Gao}^{2}$, Ya-Yi He${ }^{2}$, Cai-Cun Zhou ${ }^{2 *}$
}

\begin{abstract}
Background: Chemotherapy is the mainstay of treatment for the majority of patients with advanced nonsmall cell lung cancer (NSCLC) without driver mutations and many receive therapies beyond first-line. Secondline chemotherapy has been disappointing both in terms of response rate and survival and we know relatively little about the prognostic factors. Materials and Methods: One thousand and eight patients with advanced NSCLC who received second-line chemotherapy after progression were reviewed in Shanghai Pulmonary Hospital, China, from September 2005 to July 2010. We analyzed the effects of potential prognostic factors on the outcomes of second-line chemotherapy (overall response rate, ORR; progression free survival, PFS; overall survival, OS). Results: The response and progression free survival of first-line chemotherapy affects the ORR, PFS and OS of second-line chemotherapy (ORR: CR/PR 15.4\%, SD 10.1\%, PD2.3\%, $p<0.001$; PFS: CR/PR 3.80 months, SD 2.77 months, PD 2.03 months, $p<0.001$; OS: CR/PR 11.60 months, SD 10.33 months, PD 6.57 months, $p=0.578, p<0.001, p<0.001$, respectively). On multivariate analysis, better response to first-line therapy (CR/PR: HR=0.751, $p=0.002$; SD: HR=0.781, $p=0.021)$ and progression within 3-6 months $(\mathrm{HR}=0.626, p<0.001)$, together with adenocarcinoma $(H R=0.815, p=0.017)$, without liver metastasis $(H R=0.541, p=0.001)$, never-smoker $(\mathrm{HR}=0.772, p=0.001)$, and ECOG PS 0-1 (HR=0.745, $p=0.021)$ were predictors for good OS following secondline chemotherapy. Conclusions: Patients who responded to first-line chemotherapy had a better outcome after second-line therapy for advanced NSCLC, and the efficacy of first-line chemotherapy, period of progression, histology, liver metastasis, smoking status and ECOG PS were independent prognostic factors for OS.
\end{abstract}

Keywords: NSCLC - second-line chemotherapy - first-line chemotherapy - prognostic factor

Asian Pac J Cancer Prev, 15 (16), 6799-6804

\section{Introduction}

Lung cancer remains the leading cause of cancerrelated deaths worldwide (Ramalingam et al., 2011). Nonsmall cell lung cancer (NSCLC) accounts for about $80 \%$ of lung cancer cases and most are diagnosed at advanced stages (Lal et al., 2011; Kirmani et al., 2013). Tailored therapy based on biomarker analysis has entered the reality of lung cancer treatment (Qi et al., 2012; Gridelli et al., 2014). Patients with EGFR activated mutation or ALK/ ROS1 fusion benefit from targeted therapy with EGFR TKIs or ALK inhibitors (Gao et al., 2012). However, only a minority of patients express these markers, with EGFR mutations detected in about 30-40\% and EML4-ALK fusions in about $4 \%$ in an East Asian population (Zhou et al., 2011; Ren et al., 2012). So for the majority of patients without driver mutations, platinum-based chemotherapy remains the standard first-line treatment (Natukula et al.,
2013).

In this subpopulation, patients are recommended to receive second-line chemotherapy such as doctacel or pemetrexed when first-line treatment fails (Hanna et al., 2004; Caponi et al., 2010; Ardizzoni et al., 2012) However, the efficacy of second-line chemotherapy is still dismal, with a response rate of about $10 \%$, PFS of 2-3 months and OS of 6-9 months (Hanna et al., 2004; de Marinis et al., 2011; Ardizzoni et al., 2012). More importantly, currently, there are also still no identified predictive factors to predict the efficacy of second-line chemotherapy (Favaretto et al., 2009; de Marinis et al., 2011).

To better clarify the potential predictive factors for the outcome of second-line chemotherapy, we performed a retrospective study to investigate the association of clinical pathological features such as the efficacy of first-line chemotherapy, histology, gender and ECOG PS in 1008 Chinese patients with advanced NSCLC .

${ }^{1}$ Department of Lung Cancer and Immunology, ${ }^{2}$ Department of Medical Oncology, Shanghai Pulmonary Hospital, Tongji University Medical School Cancer Institute, Tongji University School of Medicine, People’s Republic of China ${ }^{\star}$ Equal contributors *For correspondence: caowa1990@163.com,liaiwufkyy@163.com,caicunzhoudr@163.com 


\section{Materials and Methods}

A total of 3520 patients with histologically confirmed primary stage IIIB or IV NSCLC received platinum-based doublets for first-line therapy in Shanghai Pulmonary Hospital, China from September 2005 to July 2010. Of these, 1008 patients $(28.6 \%)$ with evidence of progressive disease within 6 months after the end of first-line treatment who received second-line chemotherapy were included in our study. These 1008 patients were classified according to response to first-line chemotherapy and period of progression (Figure 1). These patients were followed up until January 1, 2013, and 784 deaths were recorded. Parameters such as efficacy of first-line chemotherapy, period of progression, gender, age, smoking history, ECOG PS, histological type, and metastasis location at diagnosis (liver, bone and brain) were chosen as potential prognostic factors for analysis in our study.

\section{Tumor evaluation and response}

Tumor response was evaluated every 6-8 weeks according to the Response Evaluation Criteria in Solid Tumors (RECIST1.1) (Nishino et al., 2010). PFS was calculated as the time between the first day of treatment and the time to documented disease progression, the last

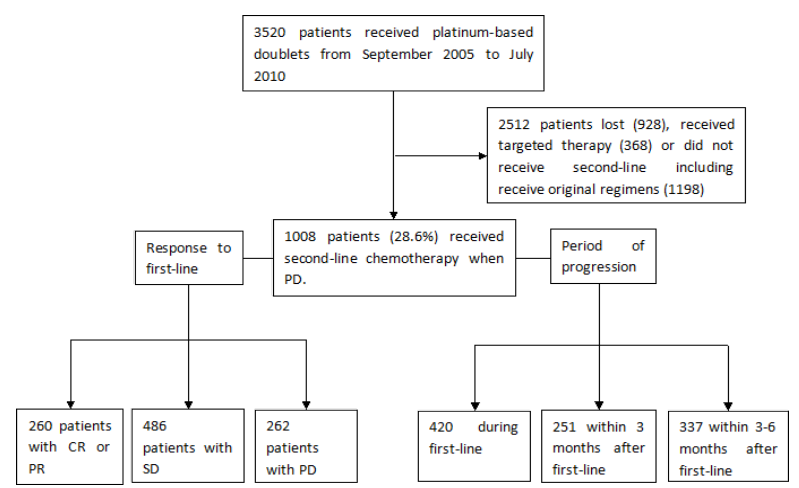

Figure 1. 3520 Patients with NSCLC; CR, complete response; $\mathrm{PR}$, partial response; $\mathrm{SD}$, stable disease; $\mathrm{PD}$, progressive disease

Table 1. Characteristics of 1008 Patients with Advanced NSCLC

\begin{tabular}{llr}
\hline Variables & & Total $\mathrm{n}=1008(\%)$ \\
\hline Gender & Male & $724(71.8 \%)$ \\
Age & Female & $284(28.2 \%)$ \\
& $<65$ years & $222(20.0 \%)$ \\
ECOG PS & $\geq 65$ years & $786(80.0 \%)$ \\
& PS=0 & $173(17.2 \%)$ \\
Smoking status & PS=1 & $749(74.3 \%)$ \\
& PS=2 & $86(8.5 \%)$ \\
Stage & Everer & $506(50.2 \%)$ \\
Histology & IIIB & $502(49.8 \%)$ \\
& IV & $282(28.0 \%)$ \\
& Squamous cell carcinoma & $726(72.0 \%)$ \\
& Adenocarcinoma & $251(24.9 \%)$ \\
Metastasis location & $635(63.0 \%)$ \\
& Adenosquamous cell carcinoma & $48(4.8 \%)$ \\
& Other & $74(7.3 \%)$ \\
& Liver & $37(3.7 \%)$ \\
& Brain & $212(21.0 \%)$ \\
& Bone & $283(28.1 \%)$ \\
& Other locations & $194(19.2 \%)$ \\
& Non-metastasis & $282(28.0 \%)$ \\
\hline
\end{tabular}

follow-up data or death from any cause. OS was calculated as the time from the start of second-line therapy to death or the last follow-up data (Zietemann et al., 2011; Di Maio et al., 2012).

\section{Statistical analysis}

The association between the efficacy of first-line treatment or related baseline factors and ORR for secondline treatment was assessed using the Chi-square test (Ardizzoni et al., 2012). The Kaplan-Meier method was used to estimate PFS and OS and the log-rank test was used to compare survival curves (Di Maio et al., 2012). A multivariate Cox-regression model was used to analyze prognostic factors for PFS and OS, presenting a hazard ratio (HR) and confidence interval (CI) for the independent variables. Statistical analyses were performed using SPSS 17.0, and a $p$-value less than 0.05 was considered statistically significant (Vergnenegre et al., 2011).

\section{Results}

\section{Characteristics of patients}

The median age of the 1008 suitable patients was 57 (range 25 to 81 ) years, with 786 patients $(80.0 \%$ ) older than 65 (elderly patients). The majority of patients were male $(71.8 \%)$, had adenocarcinoma $(63.0 \%)$ and were classed as ECOG PS 0-1 (91.5\%), and about half were never smokers $(50.2 \%)$. With regard to disease

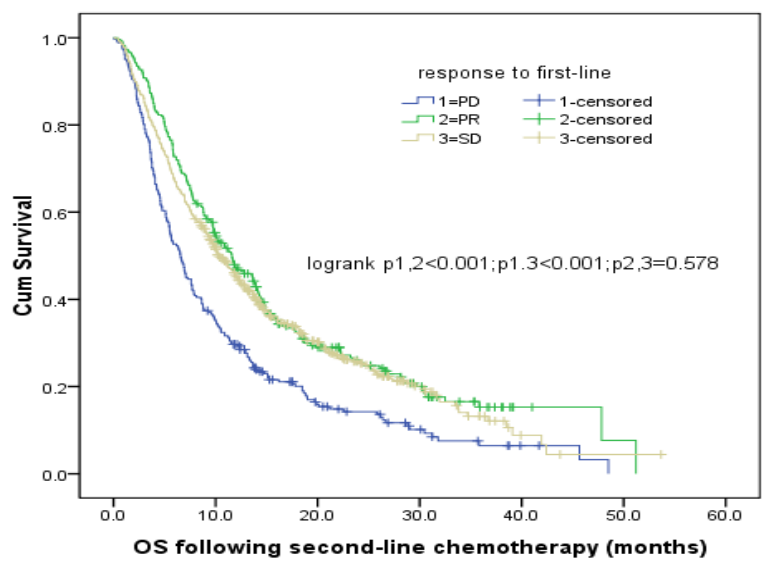

Figure 2. OS Curves for Second-Line Therapy According to the Response to First-Line Therapy

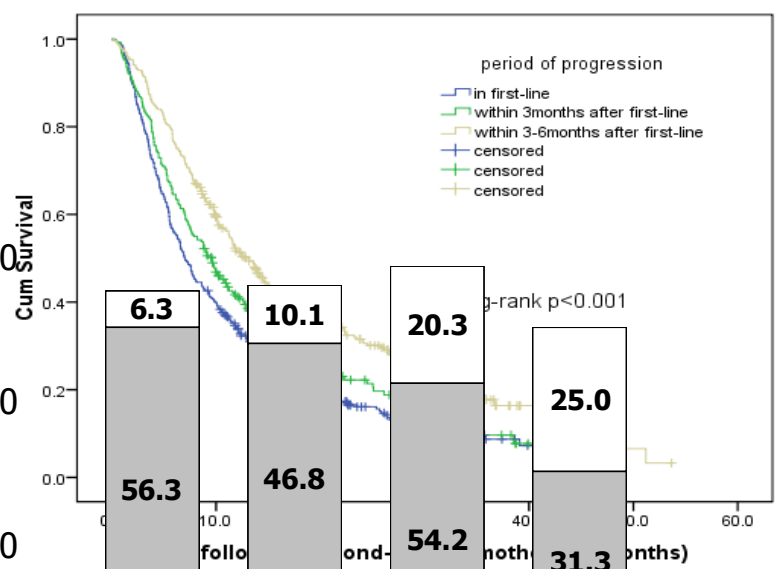

Figure 3. OS Curves for Second-Line Therapy According to Period of Progression 
Efficacy of First-line Chemotherapy Affects Second-Line Setting Response in NSCLC Patients

Table 2. The Association of Baseline Characteristics with the Survival of the 1008 Patients with Advanced NSCLC

\begin{tabular}{|c|c|c|c|c|c|}
\hline $\mathrm{N}=1008$ & & Median PFS (95\%CI) & $p$ value & Median OS $(95 \% \mathrm{CI})$ & $p$ value \\
\hline \multirow[t]{2}{*}{ Smoking status } & Never & $2.63(2.16-3.10)$ & 0.001 & $11.00(9.61-12.39)$ & $<0.001$ \\
\hline & Ever & $2.27(2.13-2.40)$ & & $7.77(6.50-9.03)$ & \\
\hline \multirow[t]{2}{*}{ Age } & $<65$ & $2.50(2.27-2.73)$ & 0.729 & $9.77(8.65-10.89)$ & 0.544 \\
\hline & $\geq 65$ & $2.37(2.21-2.53)$ & & $9.07(7.51-10.63)$ & \\
\hline \multirow[t]{2}{*}{ Gender } & Male & $2.33(2.21-2.46)$ & $<0.001$ & $8.77(7.84-9.69)$ & $<0.001$ \\
\hline & Female & $3.17(2.41-3.92)$ & & $12.93(10.84-15.03)$ & \\
\hline \multirow[t]{2}{*}{ Histology } & Squamous cell carcinoma & $2.23(2.08-2.39)$ & 0.006 & $6.97(5.80-8.14)$ & 0.001 \\
\hline & Non-squamous cell carcinoma & $2.57(2.27-2.86)$ & & $10.33(9.29-11.38)$ & \\
\hline \multirow[t]{2}{*}{ ECOG PS } & PS $0-1$ & $2.47(2.28-2.65)$ & 0.007 & $9.80(8.81-10.79)$ & 0.006 \\
\hline & PS2 & $2.13(1.86-2.41)$ & & $6.43(3.33-9.54)$ & \\
\hline \multirow[t]{2}{*}{ Liver metastasis } & Without & $2.47(2.28-2.65)$ & $<0.001$ & $9.80(8.90-10.70)$ & $<0.001$ \\
\hline & With & $1.93(1.83-2.03)$ & & $6.13(2.52-9.75)$ & \\
\hline \multirow[t]{2}{*}{ Brain metastasis } & Without & $2.43(2.26-2.60)$ & 0.688 & $9.83(8.84-10.83)$ & 0.334 \\
\hline & With & $2.40(2.10-2.70)$ & & $8.63(7.09-10.17)$ & \\
\hline \multirow[t]{2}{*}{ Bone metastasis } & Without & $2.50(2.24-2.76)$ & 0.073 & $9.83(8.75-10.92)$ & 0.126 \\
\hline & With & $2.37(2.22-2.51)$ & & $9.03(7.66-10.40)$ & \\
\hline
\end{tabular}

Table 3. Univariate and Multivariate Analysis of Prognostic Factors Regarding PFS and OS of Patients with Advanced NSCLC who Received Second-Line Chemotherapy

\begin{tabular}{|c|c|c|c|c|c|c|c|c|}
\hline \multirow[t]{3}{*}{ Variables } & \multicolumn{4}{|c|}{ PFS } & \multicolumn{4}{|c|}{ OS } \\
\hline & \multicolumn{2}{|c|}{ Univariate analysis } & \multicolumn{2}{|c|}{ Multivariate analysis } & \multicolumn{2}{|c|}{ Univariate analysis } & \multicolumn{2}{|c|}{ Multivariate analysis } \\
\hline & HR $(95 \% \mathrm{CI})$ & $\mathrm{P}$ & $\mathrm{HR}(95 \% \mathrm{CI})$ & $\mathrm{P}$ & HR $(95 \% \mathrm{CI})$ & $\mathrm{P}$ & HR $(95 \% \mathrm{CI})$ & $\mathrm{P}$ \\
\hline \multicolumn{9}{|l|}{ Histologic type } \\
\hline Squamous $v s$. non-squamous & $1.220(1.057-1.408)$ & 0.006 & $1.165(1.005-1.349)$ & 0.042 & $1.308(1.113-1.538)$ & 0.001 & $1.227(1.037-1.452)$ & 0.017 \\
\hline \multicolumn{9}{|l|}{ Period of progression } \\
\hline In first-line & 1 & & & & 1 & & & \\
\hline Within 3 months & $0.687(0.586-0.804)$ & $<0.001$ & $0.687(0.586-0.804)$ & $<0.001$ & $0.836(0.702-0.997)$ & 0.046 & $0.853(0.715-1.018)$ & 0.078 \\
\hline Within 3-6 months & $0.545(0.471-0.631)$ & $<0.001$ & $0.553(0.478-0.641)$ & $<0.001$ & $0.612(0.519-0.722)$ & $<0.001$ & $0.626(0.530-0.739)$ & $<0.001$ \\
\hline \multicolumn{9}{|l|}{ Liver metastasis } \\
\hline With $v s$. without & $2.253(1.618-3.135)$ & $<0.001$ & $2.178(1.563-3.034)$ & $<0.001$ & $1.913(1.348-2.715)$ & $<0.001$ & $1.849(1.301-2.626)$ & 0.001 \\
\hline \multicolumn{9}{|l|}{ Smoking status } \\
\hline Ever vs. never smoker & $1.221(1.078-1.382)$ & 0.002 & - & - & $1.324(1.150-1.524)$ & $<0.001$ & $1.295(1.118-1.499)$ & 0.001 \\
\hline \multicolumn{9}{|l|}{ ECOG PS } \\
\hline PS2 vs. PS0 or PS1 & $1.356(1.086-1.693)$ & 0.007 & - & - & $1.405(1.098-1.797)$ & 0.007 & $1.342(1.046-1.723)$ & 0.021 \\
\hline \multicolumn{9}{|l|}{ Efficacy of first-line therapy } \\
\hline PD & 1 & & & & 1 & & & \\
\hline CR or PR & $0.498(0.418-0.592)$ & $<0.001$ & $0.634(0.514-0.782)$ & $<0.001$ & $0.619(0.511-0.749)$ & $<0.001$ & $0.751(0.625-0.903)$ & 0.002 \\
\hline SD & $0.579(0.497-0.674)$ & $<0.001$ & $0.663(0.558-0.788)$ & $<0.001$ & $0.670(0.567-0.791)$ & $<0.001$ & $0.781(0.620-0.983)$ & 0.021 \\
\hline \multicolumn{9}{|l|}{ Gender } \\
\hline Female $v s$. male & $0.761(0.663-0.874)$ & $<0.001$ & $0.790(0.686-0.910)$ & 0.001 & $0.730(0.622-0.855)$ & $<0.001$ & - & - \\
\hline \multicolumn{9}{|l|}{ Brain metastasis } \\
\hline With $v s$. without & $1.016(0.941-1.096)$ & 0.69 & - & - & $1.043(0.958-1.136)$ & 0.335 & - & - \\
\hline \multicolumn{9}{|l|}{ Bone metastasis } \\
\hline With $v s$. without & $1.134(0.987-1.301)$ & 0.075 & - & - & $1.127(0.967-1.315)$ & 0.127 & - & - \\
\hline \multicolumn{9}{|l|}{ Age } \\
\hline$>65$ years $v s .<65$ years old & $1.022(0.902-1.159)$ & 0.731 & - & - & $1.045(0.906-1.205)$ & 0.544 & - & - \\
\hline
\end{tabular}

characteristics, $726(72.0 \%)$ patients were stage IV, and the proportion with liver, brain or bone metastasis at baseline was $3.7 \%, 21.0 \%$ and $28.1 \%$, respectively (Table 1 ).

The efficacy of first-line chemotherapy and its effect on second-line chemotherapy

The ORR and disease control rate were $25.8 \%$ and $71.4 \%$ for the first line setting, while they were $9.4 \%$ and $41.4 \%$ for the second-line chemotherapy in the 1008 patients, respectively.

The ORR for second-line therapy was $15.4 \%$ in patients who responded for first-line therapy, which was significantly higher than $10.1 \%$ in these with SD and $2.3 \%$ in these with PD $(p<0.001)$. The ORR were also significantly different according to the period of progression, which were $2.4 \%, 8.8 \%$ and $18.7 \%$ in patients who progressed on first-line therapy, within 3 months and between 3 to 6 months after the last dose of first line chemotherapy respectively $(p<0.001)$.
In terms of other clinical characteristics, the ORR of second-line chemotherapy significantly differed according to different gender (male $8.1 \% \mathrm{vs}$. female $12.7 \%, p=0.005$ ), liver metastasis (without $9.7 \% \mathrm{vs}$. with $1.0 \%, p=0.005$ ), histology type (non-squamous $9.9 \%$ vs. squamous $8.0 \%, p=0.003$ ) and smoking status (never $10.7 \%$ vs. ever smoker $8.2 \%, p=0.070$ ).

Survival analysis for second-line chemotherapy

The median PFS was 3.80 months and 2.44 months for first-line and second-line chemotherapy respectively. The median PFS and OS of second-line therapy differed significantly according to the different response status to first-line therapy. The median PFS were 3.80, 2.77 and 2.03 months in patients who response, got a stable disease and progressed from the first line chemotherapy $(p<0.001)$, while the median OS were 11.6010 .33 and 6.57 months respectively according to the different response status $(p<0.001)$ (Figure 2). In terms of period 
of progression, significant differences were also found on PFS and OS. The median PFS were 2.13, 2.63 and 4.20 months in patients who progressed on first-line therapy, within 3 months and between 3 to 6 months after the last dose of first line chemotherapy respectively $(p<0.001)$, while the median OS were 6.97, 9.57 and 13.1 months respectively according to different period of progression $(p<0.001)$ (Figure 3).

Never smokers, patients with ECOG PS 0-1, female gender, non-squamous cell carcinoma and those without liver metastasis at diagnosis had longer PFS (2.47 vs. 2.13 months, $p=0.007 ; 3.17 v s .2 .33$ months, $p<0.001 ; 2.57 v s$. 2.23 months, $p=0.006 ; 2.47$ vs. 1.93 months, $p<0.001)$. However, no differences were found between patients with and without brain or bone metastasis, or between elderly and young patients (Table 2)As for OS, patients of never smokers and female had a longer median OS after second-line therapy (11.00 vs. 7.77 months, $p<0.001$; 12.93 vs. 8.77 months, $p<0.001)$. Also, patients with nonsquamous cell carcinoma or ECOG PS 0-1 had a longer median OS than those with squamous cell carcinoma or PS 2 (10.33 vs. 6.97 months, $p=0.001 ; 9.80 v s .6 .43$ months, $p=0.006)$. Patients with liver metastasis at baseline had a worse median OS after second-line therapy (6.13 vs. 9.80 months, $p<0.001)$. However, no differences were found between elderly and young patients, or patients with and without brain or bone metastasis (Table 2).

Cox-regression model of the possible prognostic factors for PFS and OS after second-line chemotherapy

The following characteristics significantly influenced PFS after second-line therapy both by univariate and multivariate analysis: histology, period of progression, liver metastasis, the efficacy of first-line chemotherapy and gender (Table 3). Smoking status and ECOG PS were significant only by univariate analysis.

The results revealed that squamous cell carcinoma $(\mathrm{HR}=1.227, p=0.017)$, liver metastasis $(\mathrm{HR}=1.849$, $p=0.001)$, ever smoking $(\mathrm{HR}=1.295, p=0.001)$, and ECOG $\mathrm{PS} 2(\mathrm{HR}=1.342, p=0.021)$ acted as predictors of poor OS, but better efficacy of first-line therapy (CR/PR vs. PD: $\mathrm{HR}=0.751, p=0.002 ; \mathrm{SD} v s$. $\mathrm{PD}: \mathrm{HR}=0.781, p=0.021)$ and progression within 3-6 months $(\mathrm{HR}=0.626, p<0.001)$ were predictors of good OS following second-line chemotherapy. Gender was only found to have prognostic significance for OS after second-line chemotherapy in univariate analysis (female $v s$. male: $\mathrm{HR}=0.730, p<0.001$ ) (Table 3).

\section{Discussion}

In this study, we retrospectively analyses the prognosis of second-line chemotherapy in a total of 1008 patients. We found that the variables such as gender, age, smoking status, ECOG PS, liver metastasis, best efficacy of firstline therapy, and time of progression were associated with survival outcome. Among them, without liver metastasis, never smoking status, ECOG PS 0-1, non-squamous cell carcinoma, response to first-line therapy, and progression within 3-6 months after first-line chemotherapy were statistically significant associated with longer OS in multivariate analyses after second-line chemotherapy.

In terms of clinical characteristics, several previous studies have shown that different histology, smoking status, and ECOG PS could have a significantly different overall survival (Tadashi et al., 2000; Sun et al., 2010; Di Maio et al., 2012; Ali et al., 2012). In 2010, Jong-Mu Sun et al. found that female gender, adenocarcinoma, never smoking status, and ECOG PS of 0-1 were good predictive factors for subsequent pemetrexed therapy after prior gemcitabine-based chemotherapy in advanced NSCLC (Sun et al., 2010). Consistent with these reports, our study found ECOG PS 0-1 significantly associated with longer PFS and OS and numerically higher ORR. Also, patients of non-squamous cell carcinoma, never smoking status and female showed a significantly longer PFS and OS after receiving second-line chemotherapy in the univariate analysis, while female patients did not remain a predictor for longer PFS and OS in the multi-variate analysis. The EGFR mutation distribution in different subgroup might attribute to this result (Wu et al., 2010). As we all knows, it were histological type and smoking status but not gender significantly affect the incidence of EGFR mutation. Furthermore, patients with EGFR mutation showed a superiority to the efficacy of chemotherapy (Pao et al., 2010; Ren et al., 2012; Gridelli et al., 2014).

As far as we know, this study is the first one to comprehensively analysis the efficacy of first-line chemotherapy affects the response of second-line setting in 1008 patients with advanced non-small cell lung cancer. We found that best response to first-line therapy a good predictor for not only OS but also ORR and PFS following second-line chemotherapy, and the trend also existed in multivariate analysis. Furthermore, our study is the first to identify that a longer progression period as an independent positive prognostic factor for OS after second-line treatment. In line with our study, the results from several studies with limited samples suggested that the efficacy of first-line chemotherapy could affect secondline outcomes (Weiss et al., 2007; Moro-Sibilot et al., 2010; Greillier et al., 2012; Ali et al., 2012). Also, in the JMEI trial which compared pemetrexed with docetaxel as second-line setting in patients advanced NSCLC, Hanna et al found a trend that patients who had a clinical benefit from first-line therapy were more likely to benefit from second-line therapy (Hanna et al., 2004). The findings from our study, together with these previous studies suggested that the efficacy of the first line chemotherapy could be helpful to choose suitable patients for secondline chemotherapy.

Besides that, liver metastasis was known as a poor prognosis for several kinds of cancers (Zabaleta et al., 2011; Gomez et al., 2012; Ishizuka et al., 2012). Tadashi Maeda et al. found that liver metastasis was an independent prognostic factor for first-line chemotherapy in advanced NSCLC (Tadashi et al., 2000). To our knowledge, this study is the first one to investigate the effect of liver metastasis on efficacy of second-line setting in patients with NSCLC We found that liver metastasis was a strong poor predictor on PFS and OS after secondline chemotherapy. More work is needed to improve the prognosis in the NSCLC patients with liver metastasis. 
There are several limitations in this study. Firstly, we did not further analyze which might be the most beneficial agents (pemetrexed, docetaxel, or others) for patients with disease progression. Secondly, the interaction between first- and second-line drugs should also be included. Besides, for analysis of prognostic factors on efficacy of second-line treatment, we did not consider molecular markers such as TS expression, BRCA1 expression, EGFR mutation or ALK fusion, which have established roles both as determinants of treatment choice and prognostic factors.

In conclusion, patients who responded to first-line treatment had a better outcome after second-line therapy for advanced NSCLC than those who did not respond. Furthermore, the period of progression, histology, liver metastasis, smoking status and ECOG PS were independent prognostic factors for PFS and OS following second-line chemotherapy. These findings could help better evaluate the risks and benefits associated with therapeutic decisions and be used to select patients optimally for the proper choice of treatment in physicians' daily clinical practice.

\section{Acknowledgement}

The authors declare they have no conflict of interest relevant to the content of this paper.

\section{References}

Ali I, M Ali K, Mehmet K, et al (2012). Prognostic factors for second-line treatment of advanced Non-small-cell lung cancer: retrospective analysis at a single institution. Asian Pac J Cancer Prev, 13, 1281-4.

Ardizzoni A, Tiseo M, Boni L, et al (2012). Pemetrexed versus pemetrexed and carboplatin as second-line chemotherapy in advanced non-small-cell lung cancer: results of the GOIRC 02-2006 randomized phase II study and pooled analysis with the NVALT7 trial. J Clin Oncol, 30, 4501-7.

Caponi S, Vasile E, Ginocchi L, et al (2010). Second-line treatment for non-small-cell lung cancer: one size does not fit all. Clinical Lung Cancer, 11, 320-7.

de Marinis F, Ricciardi S (2011). Second-line treatment options in advanced non-small cell lung cancer. Eur J Cancer, 47, 258-71.

Di Maio M, Krzakowski M, Fougeray R, Kowalski DM, Gridelli $C$ (2012). Prognostic score for second-line chemotherapy of advanced non-small-cell lung cancer: external validation in a phase III trial comparing vinflunine with docetaxel. Lung Cancer , 77, 116-20.

Favaretto A, Pasello G, Magro C, Schettino C, Gridelli C (2009). Second and third line treatment in non-small cell lung cancer. Crit Rev Oncol Hematol, 71, 117-26.

Gridelli C, de Marinis F, Cappuzzo F, et al (2014). Treatment of advanced non-small-cell lung cancer with epidermal growth factor receptor (EGFR) mutation or ALK gene rearrangement: Results of an International Expert Panel Meeting of the Italian Association of Thoracic Oncology. Clin Lung Cancer, 15, 173-81.

Gao G, Ren S, Li A, et al (2012). Epidermal growth factor receptor-tyrosine kinase inhibitor therapy is effective as first-line treatment of advanced non-small-cell lung cancer with mutated EGFR: A meta-analysis from six phase III randomized controlled trials. Int J Cancer, 131, 822-9.

Greillier L, Barlesi F (2012). Second-line treatment of metastatic non-small cell lung carcinoma: What are the options today? What are the perspectives for tomorrow?. Rev Mal Respir, 29, 4-6 (in French).

Gomez P, Yorke R,Ayala AG, Ro JY (2012). Solid-pseudopapillary neoplasm of pancreas with long delayed liver metastasis. Ann Diagn Pathol, 16, 380-4.

Hanna N, Shepherd FA, Fossella FV, et al (2004). Randomized phase III trial of pemetrexed versus docetaxel in patients with non-small-cell lung cancer previously treated with chemotherapy. J Clin Oncol, 22, 1589-97.

Ishizuka M, Kita J, Shimoda M et al (2012). Impact of grading of liver metastasis on postoperative outcome in patients with liver metastases from colorectal cancer. Hepatogastroenterology, 59, 54-8.

Lal R, Enting D, Kristeleit H. Systemic treatment of non-smallcell lung cancer (2011). Eur J Cancer, 47, 375-7.

Moro-Sibilot D, Nishino M, Jackman DM, Hatabu H, et al (2010). New Response Evaluation Criteria in Solid Tumors (RECIST) guidelines for advanced non-small cell lung cancer: comparison with original RECIST and impact on assessment of tumor response to targeted therapy. $A J R$, 195, 221-8.

Natukula K, Jamil K, Pingali UR, et al (2013). Survival analysis in advanced non small cell lung cancer treated with platinum based chemotherapy in combination with paclitaxel, gemcitabine and etoposide. Asian Pac J Cancer Prev, 14, 4661-6.

Pao W, Chmielecki J. Rational (2010). Biologically based treatment of EGFR-mutant non-small-cell lung cancer. Nature Reviews Cancer, 10, 760-74.

Qi WX, Shen Z, Lin F et al (2012). Comparison of the efficacy and safety of EGFR tyrosine kinase inhibitor monotherapy with standard second-line chemotherapy in previously treated advanced non-small cell lung cancer: a systematic revies and meta-analysis. Asian Pac J Cancer Prev, 13, 5177-82.

Ramalingam SS, Owonikoko TK, Khuri FR (2011). Lung cancer: New biological insights and recent therapeutic advances. $C A$ Cancer J Clin, 61, 91-112.

Ren S, Kuang P, Zheng L, et al (2012). Analysis of driver mutations in female non-smoker Asian patients with pulmonary adenocarcinoma. Cell Biochem Biophys, 64, 155-60.

Ren S, Chen X, Kuang P, et al (2012). Association of EGFR mutation or ALK rearrangement with expression of DNA repair and synthesis genes in never-smoker women with pulmonary adenocarcinoma. Cancer, 118, 5588-94.

Sun JM, Oh DY, Lee SH, et al (2010). The relationship between response to previous systemic treatment and the efficacy of subsequent pemetrexed therapy in advanced non-small cell lung cancer. Lung Cancer, 68, 427-32.

Tadashi Maeda, Hiroshi U, Masahiro T, et al (2000). Prognostic factors in advanced non-small cell lung cancer: Elevated serum levels of neuron specific enolase indicate poor prognosis. Jpn J Clin Oncol, 30, 534-41.

Vergnenegre A, Corre R, Berard H, et al (2011). Costeffectiveness of second-line chemotherapy for non-small cell lung cancer: an economic, randomized, prospective, multicenter phase III trial comparing docetaxel and pemetrexed: the GFPC 05-06 study. J Thoracic Oncol, 6, 161-8.

Vergnenegre A, Smit EF, et al (2010). Second-line therapy for NSCLC in clinical practice: baseline results of the European SELECTTION observational study. Curr Med Res Opin, 26, 2661-72.

Wu M, Zhao J, Song SW, et al (2010). EGFR mutations are associated with prognosis but not with the response to front- 


\section{Wa Cao et al}

line chemotherapy in the Chinese patients with advanced non-small cell lung cancer. Lung Cancer, 67, 343-7.

Weiss GJ, Rosell R, Fossella F, et al (2007). The impact of induction chemotherapy on the outcome of second-line therapy with pemetrexed or docetaxel in patients with advanced non-small-cell lung cancer. Ann Oncol, 18, 453-60.

Zhou C, Wu Y-L, Chen G, et al (2011). Erlotinib versus chemotherapy as first-line treatment for patients with advanced EGFR mutation-positive non-small-cell lung cancer (OPTIMAL, CTONG-0802): a multicentre, openlabel, randomised, phase 3 study. Lancet Oncol, 12, 735-42.

Zietemann V, Duell T (2011). Prevalence and effectiveness of first-, second-, and third-line systemic therapy in a cohort of unselected patients with advanced non-small cell lung cancer. Lung Cancer, 73, 70-7.

Zabaleta J, Aguinagalde B, Fuentes MG, et al (2011). Survival after lung metastasectomy for colorectal cancer: Importance of previous liver metastasis as a prognostic factor. Eur J Surg Oncol, 37, 786-90. 